\title{
Intervenção Coronária Percutânea Eletiva após Fibrinólise: Dados do REMAT (Registro Madre Teresa)
}

\author{
Eduardo Cardozo Lima1, Guilherme Abreu Nascimento', Mauro Isolani Pena', \\ Alexandre von Sperling Vasconcellos', Roberto José de Queiroz Crepaldi', Walter Rabelo', \\ Roberto Luiz Marino', Sérgio Lages Murta', Ronald de Souza', Viviane Santuari Parisotto Marino', \\ Marcos Antônio Marino'
}

\section{RESUMO}

Introdução: O infarto agudo do miocárdio (IAM) permanece com elevados índices de morbidade e mortalidade e representa problema de saúde pública. Analisamos os resultados e os preditores de risco de eventos adversos hospitalares em pacientes submetidos a intervenção coronária percutânea (ICP) eletiva pós-fibrinólise. Métodos: Foram selecionados 303 pacientes com diagnóstico de IAM submetidos a reperfusão farmacológica e transferidos para um centro terciário para realização de ICP eletiva. Resultados: A população era predominantemente masculina $(76,6 \%)$, com média de idade de 59,4 \pm 11,1 anos, 18,1\% eram diabéticos e $86,8 \%$ estavam em Killip I. Estreptoquinase foi empregada em $91,7 \%$, o tempo médio de realização da ICP eletiva foi de 5,6 \pm 3,7 dias após a fibrinólise e o fluxo TIMI $3(74,2 \%)$ foi o mais prevalente. Os stents foram implantados em $97,7 \%$ dos pacientes e o sucesso angiográfico foi de $95,3 \%$. Mortalidade ocorreu em 3,3\% dos pacientes; reinfarto, em 3,6\%; revascularização da lesão-alvo, em 1,3\%; e sangramentos maiores, em $2 \%$. A análise multivariada apontou sexo feminino, idade $>65$ anos, fluxo TIMI 1, presença de trombos no vaso tratado, Killip > I e disfunção grave do ventrículo esquerdo como preditores independentes de eventos adversos hospitalares. Conclusões: A estratégia de reperfusão farmacológica seguida de transferência para realização de ICP apresenta baixas taxas de eventos adversos hospitalares e é alternativa interessante à ICP primária no cenário nacional. Necessita, no entanto, políticas públicas para aperfeiçoar a logística de manuseio desses pacientes e dispô-la de maneira eficiente a todos os hospitais de baixa e média complexidades nacionais.

DESCRITORES: Fibrinólise. Angioplastia. Stents. Infarto do miocárdio. Saúde pública.

\footnotetext{
1 Departamento de Hemodinâmica, Cardiologia e Radiologia Vascular Intervencionista - Hospital Madre Teresa - Belo Horizonte, MG, Brasil.

Correspondência: Eduardo Cardozo Lima. Av. Raja Gabaglia, $1.002-$ Bairro Gutierrez - Belo Horizonte, MG, Brasil - CEP 30380-090 E-mail: eduardocardozo@cardiol.br

Recebido em: 5/9/2011 • Aceito em: 30/11/2011
}

\section{ABSTRACT}

Elective Percutaneous Coronary Intervention after Fibrinolysis: REMAT Data (Madre Teresa Registry)

Background: Acute myocardial infarction (AMI) has a high morbidity and mortality and represents a public health problem. We analyzed the results and predictors of in-hospital adverse events in patients undergoing elective percutaneous coronary intervention $(\mathrm{PCl})$ after fibrinolysis. Methods: Three hundred and three patients with diagnosis of AMI undergoing pharmacological reperfusion and transferred to a tertiary center for elective $\mathrm{PCl}$ were selected. Results: The population included mostly men $(76.6 \%)$, with mean age of $59.4 \pm 11.1$ years, $18.1 \%$ were diabetic and $86.8 \%$ were in Killip class I. Streptokinase was used in $91.7 \%$, the mean time to perform elective $\mathrm{PCl}$ was $5.6 \pm$ 3.7 days after fibrinolysis and TIMI 3 flow was achieved in $74.2 \%$ of the patients. Stents were implanted in $97.7 \%$ and angiographic success was obtained in $95.3 \%$ of the cases. Mortality was observed in $3.3 \%$, reinfarction in $3.6 \%$, target lesion revascularization in $1.3 \%$, and major bleedings in $2 \%$ of the patients. Multivariate analysis indicated female gender, age > 65 years, TIMI 1 flow, thrombus in the treated vessel, Killip > I and severe left ventricular dysfunction were independent predictors of in-hospital adverse events. Conclusions: The pharmacological reperfusion strategy followed by transfer to perform elective $\mathrm{PCl}$ had low in-hospital adverse event rates and is an interesting alternative to primary $\mathrm{PCl}$ in Brazil. However, public policies are required to improve the logistics to better handle these patients and have them available to all low and medium complexity national hospitals.

KEY-WORDS: Fibrinolysis. Angioplasty. Stents. Myocardial infarction. Public health. 
0 infarto agudo do miocárdio (IAM), apesar dos avanços no diagnóstico e tratamento ao longo das últimas quatro décadas, permanece ainda com taxa de mortalidade considerável ${ }^{1} \mathrm{e}$, juntamente com as doenças cerebrovasculares, representa a principal causa de óbito no Brasil. ${ }^{2}$

O restabelecimento precoce da patência da artéria ocluída é o objetivo primordial do tratamento e pode ser obtido com reperfusão química ou mecânica. A reperfusão farmacológica representou avanço importante na década de 1980, com queda das taxas de mortalidade demonstrada por diversos estudos. ${ }^{3,4}$ Entretanto, as limitações inerentes à terapia fibrinolítica conduziram ao advento da intervenção coronária percutânea (ICP) primária, que rapidamente se tornou procedimento de escolha na abordagem do infarto agudo do miocárdio com supradesnivelamento do segmento ST (IAMCST), tendo em vista o comprovado maior restabelecimento de fluxo coronário epicárdico, a diminuição de complicações hemorrágicas e mecânicas, a redução das taxas de mortalidade, reinfarto e nova revascularização da lesão-alvo, além de vantagens adicionais como possibilidade de avaliação anatômica e estratificação invasiva precoce. ${ }^{5}$

Este registro tem como finalidade analisar os resultados e determinar os preditores de eventos adversos hospitalares em pacientes submetidos a ICP eletiva pós-terapia fibrinolítica com sucesso.

\section{MÉTODOS}

\section{Pacientes}

No período entre janeiro de 2004 e julho de 2011 foram selecionados 1.252 pacientes consecutivos com diagnóstico de IAMCST admitidos no Serviço de Hemodinâmica e Cardiologia Intervencionista do Hospital Madre Teresa (Belo Horizonte, MG). Foram excluídos pacientes submetidos a ICP primária e de resgate, e aqueles que apresentavam oclusão total do vaso-alvo (fluxo coronário epicárdico TIMI 0), obtendo-se como casuística final 303 pacientes submetidos a ICP eletiva pós-fibrinólise.

Os pacientes foram inicialmente atendidos em Unidades de Pronto-Atendimento (UPA) ou hospitais de baixa e média complexidades de Belo Horizonte e região metropolitana e eram conduzidos ao Laboratório de Hemodinâmica via cadastro na Central de Leitos, Sistema Único de Sáude (SUS/Fácil) e/ou contato telefônico. O diagnóstico de IAM, a seleção da terapia de reperfusão (fibrinólise ou ICP primária) e a eleição do fibrinolítico foram estabelecidos pelo centro primário.

Este estudo está em adequação com a Declaração de Helsinque no que se refere à investigação em humanos e foi aprovado pelo Comitê de Ética da instituição.

\section{Procedimento}

As ICPs foram realizadas de acordo com as diretrizes atuais e a estratégia final do procedimento foi deixada a critério do operador. Ultrassonografia intracoronária eletiva pós-ICP e filtros de proteção de embolização distal não foram empregados.

O ácido acetilsalicílico foi mantido na dose de $100 \mathrm{mg} / \mathrm{dia}$ indefinidamente e o clopidogrel $75 \mathrm{mg} / \mathrm{dia}$ foi administrado por pelo menos um mês para pacientes que receberam stents convencionais, e durante 12 meses para os tratados com stents farmacológicos. Nos pacientes que não estavam em uso de clopidogrel foi utilizada dose de ataque de $300 \mathrm{mg}$ pelo menos 24 horas antes do procedimento.

\section{Análise angiográfica e definições}

O sucesso angiográfico foi definido como lesão residual $<30 \%$, fluxo coronário epicárdico TIMI grau 3 e ausência de linhas de dissecção. Sucesso do procedimento foi definido como sucesso angiográfico na ausência de eventos cardiovasculares e cerebrovasculares adversos maiores $($ ECCAM = óbito de causa cardiovascular, acidente vascular cerebral, reinfarto, cirurgia cardíaca de emergência ou necessidade de nova revascularização percutânea do vaso-alvo). Foi considerada morte de causa cardíaca aquela decorrente de choque cardiogênico, insuficiência cardíaca, reinfarto, ruptura cardíaca, arritmia e morte súbita. ${ }^{6}$ Reinfarto foi diagnosticado na presença de alterações eletrocardiográficas (novo supradesnivelamento do segmento ST ou novas ondas Q) e/ou elevação maior que 3 vezes o valor basal de creatina quinase fração MB (CK-MB). Foi considerada cirurgia de revascularização miocárdica de emergência aquela realizada imediatamente após a ICP. Sangramento maior após ICP foi estabelecido pelos critérios do TIMI (Thrombolysis In Myocardial Infarction): presença de sangramento intracraniano ou sinais clínicos de hemorragia associada a queda dos níveis de hemoglobina $>5 \mathrm{~g} / \mathrm{dl}^{7}$

\section{Análise estatística}

A tabulação dos dados foi realizada pelo programa Microsoft Excel e a análise estatística foi realizada no software STATA versão 10. As variáveis contínuas foram expressas em média e desvio padrão; as categóricas, em números absolutos e seu porcentual. A análise univariada das variáveis categóricas foi efetivada por meio do teste qui-quadrado ou do teste exato de Fisher; variáveis contínuas foram analisadas pelo teste $t$ de Sudent ou pelo teste de Mann-Whitney, quando apropriado. Todas as variáveis com valor de $\mathrm{P}<0,2$ na análise univariada foram incluídas no modelo inicial da análise multivariada, utilizando a regressão logística, objetivando determinar os preditores independentes dos desfechos analisados. Em seguida, foram retiradas as variáveis que apresentaram maior valor de $\mathrm{P}$ até que 
restassem apenas aquelas com significância estatística $P \leq 0,05)$. Para verificação do ajuste do modelo utilizou-se o teste Hosmer-Lemeshow.

\section{RESULTADOS}

O registro apresentado aponta, em relação às características clínicas, população predominantemente masculina $(76,6 \%)$, com média de idade de 59,4 11,1 anos e 18,1\% diabéticos. À admissão, a apresentação clínica mais frequente foi a classe funcional Killip I $(86,8 \%)$ (Tabela 1$)$.

O tempo médio para a realização da ICP eletiva foi de 5,6 \pm 3,7 dias após a fibrinólise, elucidando-se que foi empregada estreptoquinase em 91,7\% dos pacientes e agentes fibrinoespecíficos (tenecteplase ou alteplase) nos demais (Tabela 1).

O levantamento dos dados angiográficos permitiu observar que os pacientes eram multiarteriais em 41,9\% dos casos, e havia disfunção ventricular global moderada a grave em 55,1\%. A artéria descendente anterior foi o vaso mais frequentemente abordado $(48,8 \%)$ e o fluxo coronário epicárdico inicial mais prevalente na artéria relacionada ao infarto foi o fluxo TIMI 3 (74,2\%) (Tabela 2).

TABELA 1

Perfil clínico dos pacientes submetidos a intervenção coronária percutânea eletiva pós-fibrinólise

\begin{tabular}{lc}
\hline Variáveis & $\mathbf{n}=\mathbf{3 0 3}$ \\
\hline Média de idade, anos & $59,4 \pm 11,1$ \\
Idade > 65 anos, n (\%) & $96(32,3)$ \\
Sexo masculino, n (\%) & $252(76,6)$ \\
Tempo médio para ICP, dias & $5,6 \pm 3,7$ \\
Hipertensão arterial, n (\%) & $221(72,9)$ \\
Diabetes, n (\%) & $55(18,1)$ \\
Dislipidemia, n (\%) & $106(35)$ \\
Tabagismo, n (\%) & $88(29)$ \\
História familiar, n (\%) & $67(21,1)$ \\
ICP prévia, n (\%) & $14(4,6)$ \\
RM prévia, n (\%) & $6(2)$ \\
Classe Killip à admissão, n (\%) & \\
I & $263(86,8)$ \\
II & $20(6,6)$ \\
III & $7(2,4)$ \\
IV & $13(4,2)$ \\
Fibrinolítico usado, n (\%) & \\
Estreptoquinase & $278(91,7)$ \\
Fibrinoespecíficos & $25(8,3)$ \\
\hline ICP = intervenção coronária percutânea; n & $=$ número de \\
pacientes; RM = cirurgia de revascularização do miocárdio. \\
\hline
\end{tabular}

Os stents foram implantados em 97,7\% dos pacientes, sendo a minoria stents farmacológicos. Foi necessário o uso de balão intra-aórtico em 4,2\% dos pacientes em decorrência de choque cardiogênico. A terapia adjunta farmacológica com inibidores da glicoproteína IIb/IIla (abciximab ou tirofiban) foi praticada em 10,6\%. Sucesso angiográfico da ICP foi alcançado em $95,3 \%$ dos pacientes e sucesso clínico, em 87,1\%.

A avaliação de incidência de complicações maiores na fase hospitalar demonstra taxas de mortalidade de $3,3 \%$ e de reinfarto de $3,6 \%$, e necessidade de nova revascularização de lesão-alvo de 1,3\% (Tabela 3). Sangramentos maiores ocorreram em $2 \%$ dos pacientes. Não ocorreu acidente vascular cerebral nem houve necessidade de cirurgia de revascularização de urgência.

A análise multivariada elucidou os preditores independentes de insucesso: ECCAM e sangramento maior na fase hospitalar (Tabela 4). Nota-se que o sexo feminino foi preditor de insucesso do procedimento, óbito e sangramento maior e idade > 65 anos foi preditora de insucesso e sangramento maior. O fluxo coronário epicárdico TIMI 1 foi preditor de insucesso, óbito e reinfarto. A presença de trombos no vaso tratado constituiu fator de risco que elevou os desfechos insucesso

TABELA 2

Perfil angiográfico e relacionado ao procedimento dos pacientes submetidos a intervenção coronária percutânea eletiva pós-fibrinólise

\begin{tabular}{|c|c|}
\hline Variáveis & $n=303$ \\
\hline Doença multiarterial, n (\%) & $127(41,9)$ \\
\hline $\begin{array}{l}\text { Disfunção ventricular } \\
\text { moderada/grave, n (\%) }\end{array}$ & $167(55,1)$ \\
\hline \multicolumn{2}{|l|}{ Vaso tratado, n (\%) } \\
\hline DA & $148(48,8)$ \\
\hline CD & $133(43,9)$ \\
\hline $\mathrm{Cx}$ & $20(6,6)$ \\
\hline Enxerto de safena & $2(0,7)$ \\
\hline \multicolumn{2}{|l|}{ Fluxo epicárdico coronário inicial, n (\%) } \\
\hline TIMI 1 & $67(22,1)$ \\
\hline TIMI 2 & $11(3,7)$ \\
\hline TIMI 3 & $225(74,2)$ \\
\hline Trombos no vaso tratado, n (\%) & $64(21,1)$ \\
\hline $\begin{array}{l}\text { Uso de inibidores de } \\
\text { glicoproteína IIb/IIla, n (\%) }\end{array}$ & $32(10,6)$ \\
\hline Implante de balão intra-aórtico, n (\%) & $13(4,2)$ \\
\hline Implante de stent, n (\%) & $296(97,7)$ \\
\hline Stent convencional & $277(93,6)$ \\
\hline Stent farmacológico & $19(6,4)$ \\
\hline Sucesso do procedimento, n (\%) & $264(87,1)$ \\
\hline
\end{tabular}


e óbito. As classes de apresentação clínica Killip > I consolidaram-se como preditoras independentes para todos os eventos adversos examinados.

\section{DISCUSSÃO}

Os dados apresentados neste estudo observacional transversal de mundo real revelam importantes aspectos acerca do perfil e da evolução dos pacientes com quadro de IAMCST submetidos à estratégia de fibrinólise com critérios de reperfusão e encaminhados para realização de ICP eletiva em um centro de referência de alta complexidade em cardiologia na região metropolitana de Belo Horizonte.

TABELA 3

Eventos adversos maiores na fase hospitalar

\begin{tabular}{lc}
\hline Variáveis & $\mathbf{n}=\mathbf{3 0 3}$ \\
\hline Mortalidade, n (\%) & $10(3,3)$ \\
AVC, n (\%) & 0 \\
Reinfarto, n (\%) & $11(3,6)$ \\
Cirurgia de emergência, n (\%) & 0 \\
Nova RLA, n (\%) & $4(1,3)$ \\
Sangramento maior, n (\%) & $6(2)$ \\
\hline AVC = acidente vascular cerebral; $\mathrm{n}=$ número de pacientes; \\
RLA = revascularização da lesão-alvo \\
\hline
\end{tabular}

Verifica-se que as informações referentes ao perfil clínico da população examinada, incluindo idade, sexo e fatores de risco tradicionais para aterosclerose, são concordantes com as observadas em outros estudos ${ }^{8,9}$, especialmente aqueles publicados com base no banco de dados da Central Nacional de Intervenções Cardiovasculares da Sociedade Brasileira de Hemodinâmica e Cardiologia Intervencionista (CENIC). Cabe ressaltar a elevada proporção de pacientes $(91,7 \%$ ) que ainda são submetidos a fibrinólise com estreptoquinase, apesar das evidências científicas favoráveis aos trombolíticos fibrinoespecíficos ${ }^{10}$, informação similar apresentada em registro nacional. ${ }^{9}$ Esse cenário certamente é determinado por questões de ordem financeira, uma vez que a estreptoquinase é consideravelmente mais barata que os demais fibrinolíticos.

Do mesmo modo, os dados angiográficos encontrados também são condizentes com trabalhos já publicados, sendo fundamental destacar que, neste registro, cerca de 98\% dos pacientes admitidos após fibrinólise bem-sucedida exibiam à coronariografia estenose residual $>50 \%$ na artéria relacionada ao IAM, e quase metade era portadora de doença multiarterial, constatações que justificam a estratificação invasiva eletiva nesse grupo de pacientes.

A análise dos resultados imediatos demonstra o emprego de stents na ICP de praticamente todos os pacientes, e, neste grupo, a predominância do uso de stents convencionais $(93,6 \%)$, fato provavelmente jus-

TABELA 4

Preditores independentes de insucesso e de eventos adversos maiores na fase hospitalar

\begin{tabular}{|c|c|c|c|}
\hline Evento & Variáveis & Valor de $\mathbf{P}$ & OR (IC 95\%) \\
\hline \multirow[t]{5}{*}{ Insucesso } & Sexo feminino & 0,002 & $3,51(1,19-10,39)$ \\
\hline & Idade $>65$ anos & 0,043 & $5,31(1,06-70,8)$ \\
\hline & Classe Killip > I & 0,015 & $4,28(1,32-13,87)$ \\
\hline & Fluxo TIMI 1 & 0,017 & $4,42(1,3-14,98)$ \\
\hline & Trombo no vaso tratado & $<0,001$ & $16,32(4,4-60,56)$ \\
\hline \multirow[t]{6}{*}{ Mortalidade } & Diabetes & 0,015 & $4,86(1,36-17,42)$ \\
\hline & Sexo feminino & 0,034 & $5,23(1,13-24,27)$ \\
\hline & Trombo no vaso tratado & 0,034 & $3,97(1,11-14,15)$ \\
\hline & Fluxo TIMI 1 & 0,027 & $4,25(1,19-17,46)$ \\
\hline & Classe Killip > I & $<0,001$ & $11,42(3,07-42,55)$ \\
\hline & Disfunção moderada a grave & 0,019 & $13,03(2,48-51,04)$ \\
\hline \multirow[t]{3}{*}{ Reinfarto } & Disfunção moderada a grave & 0,015 & $2,48(1,51-12)$ \\
\hline & Classe Killip > I & 0,031 & $4,06(1,13-14,57)$ \\
\hline & Fluxo TIMI 1 & 0,018 & $8,14(1,44-46,1)$ \\
\hline \multirow[t]{3}{*}{ Sangramento maior } & Idade $>65$ anos & 0,047 & $1,08(1,01-1,17)$ \\
\hline & Sexo feminino & 0,028 & $6,86(1,23-38,31)$ \\
\hline & Classe Killip > I & 0,045 & $7,03(1,36-36,11)$ \\
\hline
\end{tabular}

IC 95\% = intervalo de confiança de 95\%; ICP = intervenção coronária percutânea; OR = odds ratio. 
tificado pela falta de evidências em relação à redução de mortalidade e reinfarto dos stents farmacológicos na fase aguda do IAM e por questões relativas ao maior impacto financeiro imediato, o que motiva o SUS e a maioria das operadoras de saúde suplementar a não autorizá-los.

Quanto à análise dos desfechos maiores, a exemplo de publicações nacionais ${ }^{9-11}$, nota-se elevado índice de sucesso do procedimento (com porcentual próximo a $90 \%$ ) e baixo porcentual de eventos adversos, fato atribuído à maior experiência acumulada dos cardiologistas invasivos do Serviço de Hemodinâmica, visto que curva de aprendizado e volume de procedimentos influenciam decisivamente os resultados da ICP, como divulgado por relevante registro americano. ${ }^{12} \mathrm{Em}$ especial, salienta-se baixa taxa de mortalidade $(3,3 \%)$, comparável à encontrada em ensaios internacionais, como a metanálise publicada por Collet et al. ${ }^{13}(3,8 \%)$, ressalvando-se que nos ensaios internacionais os fibrinolíticos mais utilizados foram alteplase ou reteplase e o tempo médio de realização da ICP foi extremamente precoce. Não há informações nacionais disponíveis publicadas acerca da mortalidade na ICP após fibrinólise bem-sucedida. No trabalho brasileiro relatado por Mattos et al. ${ }^{9}$ e fundamentado em dados da CENIC, depara-se com mortalidade de 7,4\% nas ICPs primárias e de 5,6\% nas ICPs de resgate. Ademais, ressalta-se a não ocorrência de acidentes vasculares cerebrais e de necessidade de cirurgia cardíaca de urgência, em linha com tendências atuais da ICP.

A análise multivariada possibilitou identificar possíveis preditores de insucesso da ICP, ocorrência de ECCAM e sangramento maior. A relação das variáveis disfunção ventricular grave, sexo feminino, diabetes, classe Killip > I e fluxo epicárdico inicial com a taxa de mortalidade após o IAM era esperada e também é consoante com a literatura. ${ }^{14}$

Ressalta-se que o tempo médio apurado de realização da ICP eletiva após o fibrinolítico, de 5,6 dias, pode ser considerado muito elevado, principalmente quando comparado ao preconizado por ensaios nos últimos anos. ${ }^{11,15,16}$ Recente publicação ${ }^{17}$ refere tempo médio em centros brasileiros ainda maior (8,6 dias), realidade nacional desafiadora e que certamente exige políticas públicas para adequar a logística de encaminhamento de pacientes pós-fibrinólise de UPA e hospitais primários para centros terciários.

A ICP eletiva sistemática era, até recentemente, contraindicada em pacientes submetidos a fibrinólise e sem evidência de isquemia miocárdica, especialmente porque os estudos comparativos não empregavam o implante de stents. Contudo, evidências consistentes atuais obtidas a partir de trabalhos bem conduzidos $^{11,15,16,18-21}$ e com a incorporação do uso de stents demonstram a eficácia e a segurança da ICP eletiva e precoce (preferencialmente inferior a 24 horas, e com possível benefício até 72 horas após a fibrinólise bem-sucedida), com redução significativa das taxas de morte e reinfarto, quando comparadas à estratégia conservadora guiada pela presença de sintomas ou isquemia induzida em testes funcionais. A comparação com atrasos superiores a 72 horas ainda não foi estabelecida. A Sociedade Europeia de Cardiologia, amparada nesses subsídios, elevou essa estratégia terapêutica a classe de recomendação I, nível de evidência A em diretriz sobre revascularização miocárdica ${ }^{22}$ publicada em 2010. As Diretrizes da Sociedade Brasileira de Cardiologia Intervenção Coronária Percutânea e Métodos Adjuntos Diagnósticos em Cardiologia Intervencionista (2008) ${ }^{23}$ ainda mantêm a classe de recomendação Ila, com retardo aceitável de até 96 horas para o intervalo fibrinólise-ICP, sendo aguardada modificação para classe I.

Visto que as doenças cardiovasculares são a maior causa de morte no Brasil e no mundo, com geração de importante impacto econômico para a sociedade de um modo geral, e que sua ascensão pode ser controlada, há de se destacar a iniciativa do Ministério da Saúde, que, recentemente, lançou plano de ações para o enfrentamento das doenças crônicas não-transmissíveis no Brasil. ${ }^{24}$ Foram propostas metas e estratégias que pretendem atenuar o panorama atual, como qualificar o atendimento ao IAM nas urgências pré-hospitalares (Serviço de Atendimento Móvel de Urgência - SAMU e UPA), utilizar métodos de telemedicina que permitam diagnóstico precoce e preciso do IAM, implantação de protocolos para agilizar a transferência de pacientes infartados, ampliar o acesso à ICP primária, definir normas para a realização de fibrinólise no IAM, expandir os leitos destinados ao tratamento dos infartados, e divulgar informações referentes à identificação precoce do IAM, medidas que certamente vêm ao encontro dos anseios dos cardiologistas clínicos e intervencionistas e de toda a população.

\section{Limitações do estudo}

São limitações deste estudo a análise retrospectiva dos dados, sua realização em um único centro e a ausência de seguimento tardio. A análise multivariada detectou os prováveis preditores independentes de eventos adversos, mas em decorrência da amplitude dos intervalos de confiança, consequente à baixa frequência dos eventos analisados, pouco se pode afirmar a respeito da força de associação entre essas variáveis e os desfechos analisados.

\section{CONCLUSÕES}

Neste registro, verificou-se alto índice de sucesso e baixa ocorrência de eventos adversos hospitalares nos pacientes pós-fibrinólise. Averiguou-se ainda que o tempo médio de realização da ICP após a terapia fibrinolítica é alto e é inquestionável a necessidade de adotar medidas que facilitem o acesso precoce desses pacientes a um centro terciário. 
Enfatiza-se que a ICP primária deve ser sempre indicada para todos os pacientes elegíveis no tratamento do IAMCST. Porém, partindo-se do pressuposto de que essa estratégia terapêutica ainda é pouco factível em nosso País e, inclusive, em muitos países desenvolvidos, recomenda-se proceder rápida reperfusão farmacológica, preferencialmente com trombolíticos fibrinoespecíficos, acompanhada de transferência precoce e sistemática para centro habilitado para realização de ICP.

\section{CONFLITO DE INTERESSES}

Os autores declaram não haver conflito de interesses relacionado a este manuscrito.

\section{REFERÊNCIAS}

1. Canto JG, Rogers WJ, Chandra NC, French WJ, Barron HV, Frederick PD, et al. The association of sex and payer status on management and subsequent survival in acute myocardial infarction. Arch Intern Med. 2002;162(5):587-93.

2. Brasil. Ministério da Saúde. DATASUS. Informações de saúde [Internet]. [citado 2011 set. 30]. Disponível em: http://www2. datasus.gov.br/DATASUS/index.php?area $=02$

3. Gruppo Italiano per lo Studio dela Streptochinasi nell'Infarto Miocardico (GISSI). Effectiveness of intravenous thrombolytic treatment in acute myocardial infarction. Lancet. 1986;1(8478): 397-402.

4. Fibrinolytic Therapy Trialists (FTT) Collaborative Group. Indications for fibrinolytic therapy in suspected acute myocardial infarction: collaborative overview of early mortality and major morbidity results from all randomised trial of more than 1000 patients. Lancet. 1994;343(8893):311-22.

5. Keeley EC, Boura JA, Grines CL. Primary angioplasty versus intravenous thrombolytic therapy for acute myocardial infarction: a quantitative review of 23 randomised trials. Lancet. 2003; 361 (9351):13-20.

6. King SB $3^{\text {rd }}$, Smith SC Jr, Hirshfeld JW Jr, Jacobs AK, Morrison DA, Williams DO, et al. 2007 Focused Update of the ACC/ AHA/SCAI 2005 Guideline Update for Percutaneous Coronary Intervention: a report of the American College of Cardiology/ American Heart Association Task Force on Practice Guidelines: 2007 Writing Group to Review New Evidence and Update the ACC/AHA/SCAI 2005 Guideline Update for Percutaneous Coronary Intervention, Writing on Behalf of the 2005 Writing Committee. Circulation. 2008;117(2):261-95.

7. TIMI Study Group. Definitions used in TIMI trials [Internet]. [cited 2011 Sept 30]. Available from: http://www.timi.org/ wp-content/uploads/2010/10/TIMI-Definitions.pdf

8. Mattos LA, Zago A, Chaves A, Pinto I, Tanajura L, Staico R, et al. Revascularização coronariana percutânea primária no infarto agudo do miocárdio: análise comparativa dos resultados imediatos em pacientes progressivamente longevos. Arq Bras Cardiol. 2001;76(1):53-62.

9. Mattos LA, Sousa AGMR, Pinto IMF, Silva ER, Carneiro JK, Sousa JE, et al. Uma comparação entre a intervenção coronariana percutânea de resgate e primária realizadas no infarto agudo do miocárdio: relato multicêntrico de 9.371 pacientes. Arq Bras Cardiol. 2004;82(5):434-9.

10. GUSTO Investigators. An international randomized trial comparing four thrombolytic strategies for acute myocardial infarction. N Engl J Med. 1993;329(10):673-82.

11. Fernandez-Aviles F, Alonso JJ, Castro-Beiras A, Vázquez N, Blanco J, Alonso-Briales J, et al. Routine invasive strategy within 24 hours of thrombolysis versus ischaemia-guided conservative approach for acute myocardial infarction with ST-segment elevation (GRACIA-1): a randomised controlled trial. Lancet. 2004;364(9439):1045-53.

12. Rogers WJ, Canto JG, Lambrew CT, Tiefenbrunn AJ, Kinkaid B, Shoultz DA, et al. Temporal trends in the treatment of over 1,5 million patients with myocardial infarction in the USA from 1990 through 1999. J Am Coll Cardiol. 2000;36(7):2056-63.

13. Collet JP, Montalescot G, Le May M, Borentain M, Gershlick A. Percutaneous coronary intervention after fibrinolysis: a multiple meta-analyses approach according to the type of strategy. J Am Coll Cardiol. 2006;48(7):1326-35.

14. Stone GW, Grines CL, Browne KF, Marco J, Rothbaum D, O'Keefe J, et al. Predictors of in-hospital and 6-month outcome after acute myocardial infarction in the reperfusion era: the Primary Angioplasty in Myocardial Infarction (PAMI) trail. J Am Coll Cardiol. 1995;25(2):370-7.

15. Di Mario C, Dudek D, Piscione F, Mielecki W, Savonitto S, Murena E, et al. Immediate angioplasty versus standard therapy with rescue angioplasty after thrombolysis in the Combined Abciximab REteplase Stent Study in Acute Myocardial Infarction (CARESS-in-AMI): an open, prospective, randomised, multicentre trial. Lancet. 2008;371(9612):559-68.

16. Cantor WJ, Fitchett D, Borgundvaag B, Heffernan M, Cohen EA, Morrison LJ, et al. Rationale and design of the Trial of Routine Angioplasty and Stenting After Fibrinolysis to Enhance Reperfusion in Acute Myocardial Infarction (TRANSFER-AMI). Am Heart J. 2008;155(1):19-25.

17. Matte BS, Bergoli LCC, Balvedi JA, Zago AC. Perfil da intervenção coronária percutânea no infarto agudo do miocárdio com supradesnivelamento do segmento ST no Brasil de 2006 a 2010: Registro CENIC. Rev Bras Cardiol Invasiva. 2011; 19(2):131-7.

18. Cantor WJ, Fitchett D, Borgundvaag B, Ducas J, Heffernan M, Cohen EA, et al. Routine early angioplasty after fibrinolysis for acute myocardial infarction. N Engl J Med. 2009;360(26): 2705-18.

19. D'Souza SP, Mamas MA, Fraser DG, Fath-Ordoubadi F. Routine early coronary angioplasty versus ischaemia-guided angioplasty after thrombolysis in acute ST-elevation myocardial infarction: a meta-analysis. Eur Heart J. 2010;32(8):972-82.

20. Sánchez PL, Fernández-Avilés F. Routine early coronary angioplasty after thrombolysis in acute ST-elevation myocardial infarction: lysis is not the final step. Eur Heart J. 2011;32(8): 927-30.

21. Stone GW. Angioplasty strategies in ST-segment-elevation myocardial infarction: part II: intervention after fibrinolytic therapy, integrated treatment recommendations, and future directions. Circulation. 2008;118(5):552-66.

22. Wijns $W$, Kolh $P$, Danchin N, Di Mario C, Falk V, Folliguet $\mathrm{T}$, et al.; The Task Force on Myocardial Revascularization of the European Society of Cardiology (ESC) and the European Association for Cardio-Thoracic Surgery (EACTS). Guidelines on myocardial revascularization. Eur Heart J. 2010;31(20): 2501-55.

23. Mattos LA, Lemos Neto PA, Rassi A Jr, Marin-Neto JA, Sousa AGMR, Devito FS, et al. Diretrizes da Sociedade Brasileira de Cardiologia - Intervenção Coronária Percutânea e Métodos Adjuntos Diagnósticos em Cardiologia Intervencionista (II Edição - 2008). Arq Bras Cardiol. 2008;91(4 Supl 1):1-58.

24. Brasil. Ministério da Saúde; Secretaria de Vigilância em Saúde. Plano de Ações Estratégicas para o Enfrentamento das Doenças Crônicas Não-Transmissíveis (DCNT) no Brasil, 2011-2012 [Internet]. Brasília; 2011 [citado 2011 out. 13]. Disponível em: http://portal.saude.gov.br/portal/arquivos/pdf/cartilha plano final_11.pdf 